\title{
Criminologie
}

\section{Personnalité et distorsions cognitives des agresseurs sexuels} Changements longitudinaux à la suite d'un traitement pour agression sexuelle envers les enfants

\section{Personality and cognitive distortions in sexual offenders} Longitudinal changes after treatment for child sexual abuse

Personalidad y distorsiones cognitivas de los agresores

sexuales

\author{
Cambios longitudinales después de un tratamiento por \\ agresión sexual hacia los niños
}

\section{Marie-Ève Daspe, Yvan Lussier, Valérie Desaulniers, Natacha Godbout, Alain Perron, Stéphane Sabourin et Francine Bronsard}

Volume 50, numéro 1, printemps 2017

L'agression sexuelle commise sur des mineurs : les victimes, les auteurs

URI : https://id.erudit.org/iderudit/1039803ar

DOI : https://doi.org/10.7202/1039803ar

Aller au sommaire du numéro

Éditeur(s)

Les Presses de l’Université de Montréal

ISSN

0316-0041 (imprimé)

1492-1367 (numérique)

Découvrir la revue

Citer cet article

Daspe, M.-È., Lussier, Y., Desaulniers, V., Godbout, N., Perron, A., Sabourin, S. \& Bronsard, F. (2017). Personnalité et distorsions cognitives des agresseurs sexuels : changements longitudinaux à la suite d'un traitement pour agression sexuelle envers les enfants. Criminologie, 50(1), 233-262.

https://doi.org/10.7202/1039803ar

\section{Résumé de l'article}

Bien qu'il n'existe pas de typologie définitive des caractéristiques des agresseurs sexuels à l'égard d'enfants, la documentation scientifique révèle la présence de distorsions cognitives et de traits de personnalité impliqués dans l'étiologie et le maintien des comportements sexuels déviants. Ces facteurs étant également associés au risque de récidive, ils constituent des cibles de traitement de première importance. La présente étude a pour objectif d'examiner l'évolution des distorsions cognitives et des traits de personnalité d'hommes en traitement pour agressions sexuelles à l'égard d'enfants. L'échantillon est composé de 134 agresseurs sexuels intrafamiliaux, recrutés à leur entrée au Programme d'évaluation et de traitement des agressions sexuelles (PÉTAS). Ceux-ci sont évalués à trois reprises, soit à leur entrée au PÉTAS, ainsi qu'à la fin de la première et de la deuxième année de traitement. Les résultats montrent des diminutions significatives sur l'ensemble des distorsions cognitives de nature sexuelle, une diminution du névrosisme et une augmentation de l'extraversion entre les temps de mesure. Sur le plan individuel, des proportions importantes d'hommes présentent des améliorations sur la majorité des variables mesurées. Ces résultats soutiennent la malléabilité des distorsions cognitives et de certains traits de personnalité et appuient l'efficacité du PÉTAS. 


\title{
Personnalité et distorsions cognitives des agresseurs sexuels
}

\author{
Changements longitudinaux à la suite d'un \\ traitement pour agression sexuelle envers \\ les enfants
}

\author{
Marie-Ève Daspe \\ Université du Québec à Montréal \\ daspe.marie-eve@courrier.uqam.ca \\ Yvan Lussier \\ Université du Québec à Trois-Rivières \\ yvan.lussier@uqtr.ca

\section{Valérie Desaulniers} \\ Université du Québec à Trois-Rivières \\ valerie.desaulniers1@uqtr.ca \\ Natacha Godbout \\ Université du Québec à Montréal \\ godbout.natacha@uqam.ca
}

\author{
Alain Perron \\ Psychologue, pratique privée \\ alain.perron.2@cgocable.ca \\ Stéphane Sabourin \\ Université Laval \\ stephane.sabourin@psy.ulaval.ca \\ Francine Bronsard \\ Centre jeunesse de la Mauricie et \\ du Centre-du-Québec \\ Francine_Bronsard@ssss.gouv.qc.ca
}

RÉSUMÉ - Bien qu'il n'existe pas de typologie définitive des caractéristiques des agresseurs sexuels à l'égard d'enfants, la documentation scientifique révèle la présence de distorsions cognitives et de traits de personnalité impliqués dans l'étiologie et le maintien des comportements sexuels déviants. Ces facteurs étant également associés au risque de récidive, ils constituent des cibles de traitement de première importance. La présente étude a pour objectif d'examiner l'évolution des distorsions cognitives et des traits de personnalité d'hommes en traitement pour agressions sexuelles à l'égard d'enfants. L'échantillon est composé de 134 agresseurs sexuels intrafamiliaux, recrutés à leur entrée au Programme d'évaluation et de traitement des agressions sexuelles (PÉTAS). Ceux-ci sont évalués à trois reprises, soit à leur entrée au PÉTAS, ainsi qu'à la fin de la première et de la deuxième année de traitement. Les résultats montrent

1. Marie-Ève Daspe est maintenant à l'University of Southern California, Department of Psychology, SGM 930, University Park Campus, Los Angeles, CA 90089-1061. Pour toute correspondance, veuillez communiquer avec Yvan Lussier: yvan.lussier@uqtr.ca. 
des diminutions significatives sur l'ensemble des distorsions cognitives de nature sexuelle, une diminution du névrosisme et une augmentation de l'extraversion entre les temps de mesure. Sur le plan individuel, des proportions importantes d'hommes présentent des améliorations sur la majorité des variables mesurées. Ces résultats soutiennent la malléabilité des distorsions cognitives et de certains traits de personnalité et appuient l'efficacité du PÉTAS.

MotS CLÉS - Agresseurs sexuels, distorsions cognitives, personnalité, traitement.

\section{Introduction}

Les agressions sexuelles constituent une problématique majeure au sein de la société québécoise. Il est estimé que près d'une femme sur quatre et un homme sur dix seront victimes d'un délit sexuel avant d'atteindre la majorité (Hébert, Tourigny, Cyr, McDuff et Joly, 2009). De surcroît, les répercussions sur les victimes demeurent préoccupantes: symptômes dissociatifs, stress post-traumatique, troubles de l'humeur, comportements suicidaires, comportements sexuels à risque, abus de substances, etc. (voir Collin-Vézina, Daigneault et Hébert, 2013, pour une revue de ces écrits). Afin de prévenir les agressions sexuelles perpétrées à l'endroit de jeunes, il importe de cerner, chez les agresseurs, les facteurs associés aux délits commis. Diverses caractéristiques telles que les distorsions cognitives soutenant les agressions sexuelles ainsi que certains traits de personnalité expliquent partiellement ces gestes (Abel, Becker et Cunningham-Rathner, 1984; Becerra-García, García-León, Muela-Martínez et Egan, 2013). Cet article examine les changements sur le plan de ces facteurs de risque chez des agresseurs sexuels intrafamiliaux à la suite d'un traitement spécifique aux agressions sexuelles d'enfants.

\section{Agresseurs sexuels}

Les hommes sont surreprésentés dans les délits sexuels (Douglas et Finkelhor, 2005; Ogrodnik, 2010) et constituent la majorité des agresseurs sexuels envers les garçons (83\%) et les filles (98\%) (Finkelhor, Hotaling, Lewis et Smith, 1990). Par ailleurs, près du tiers des agressions sexuelles à l'enfance sont commises par un membre de la famille (Douglas et Finkelhor, 2005). Les agressions sexuelles intrafamiliales sont donc principalement perpétrées par un homme de la famille élargie (grand-père, oncle, cousin, etc.) ou immédiate (p. ex., père biologique, beau-père ou frère) (Ogrodnik, 2010; Tourigny, Daigneault, 
Hébert et Wright, 2005; Trocmé et al., 2001). Outre ces caractéristiques, les personnes commettant des agressions sexuelles à l'égard d'enfants constituent une population diversifiée (Robertiello et Terry, 2007). Certains auteurs ont tenté de développer des typologies fondées, par exemple, sur les préférences sexuelles, la personnalité, la présence de psychopathologies ou les types de délinquants sexuels (Beech, 1998; Furniss, 1987; Guay, Proulx et Ouimet, 2001; Marshall, Barbaree et Christophe, 1986; Scott et Stone, 1986). Cependant, plusieurs individus ne se retrouvent pas dans ces catégories discrètes (Robertiello et Terry, 2007), les facteurs menant à des infractions sexuelles incluant une combinaison complexe d'éléments biologiques, psychologiques, interpersonnels, socioculturels et institutionnels.

\section{Efficacité et cibles des traitements}

En dépit de la rareté des études cliniques randomisées soutenant l'efficacité des traitements, les méta-analyses disponibles semblent indiquer une réduction de la récidive sexuelle variant de $27 \%$ à $37 \%$, en réaction à des interventions propres aux agressions sexuelles (Hanson et al., 2002; Lösel et Schmucker, 2005). Sur le plan de l'évaluation de la récidive, deux catégories de facteurs sont considérées. Les facteurs de risque statiques, tels que l'âge, le sexe et l'histoire criminelle (Andrews, Bonta et Wormith, 2006) sont évalués à l'aide d'outils actuariels (p. ex., Hanson et Thornton, 1999) qui prédisent avec une grande précision le risque de récidive (Hanson et Morton-Bourgon, 2009). Cependant, ces facteurs ne sont pas toujours associés au contexte psychologique et social et ne peuvent être la cible d'un effort de changement (Yesberg et Polaschek, 2015). Les facteurs de risque dynamiques, ou besoins criminogènes (Andrews et Bonta, 2010), réfèrent aux attitudes, difficultés relationnelles, problèmes d'autorégulation, etc., qui soutiennent l'agression sexuelle (Beech, Fisher et Thornton, 2003; Helmus, Hanson, Babchishin et Mann, 2013). Contrairement aux facteurs statiques, les facteurs dynamiques présentent un potentiel de changement et fournissent des estimations plus ponctuelles du risque de récidive (Hanson et Morton-Bourgon, 2009). Ces derniers constituent donc les cibles d'intervention centrales des traitements offerts aux agresseurs sexuels (Murphy, 2004). Les attitudes et les distorsions cognitives soutenant les délits sexuels (Helmus et al., 2013) et, dans une moindre mesure, la personnalité (Craig, Thornton, Beech et Browne, 2007; Hanson et 
Harris, 2000) sont des facteurs dynamiques qui ressortent de la documentation scientifique et qui sont examinés dans le cadre de cette recherche.

\section{Distorsions cognitives et croyances erronées}

Les distorsions cognitives sont définies comme un ensemble de croyances, d'attitudes et d'affirmations erronées (Abel et al., 1984; Murphy, 1990) employées pour justifier les actes perpétrés (Segal et Stermac, 1990). En se fondant sur la théorie de l'apprentissage social (Bandura, 1977), Abel et ses collaborateurs (1984) proposent que les distorsions cognitives des agresseurs sexuels visent à se désengager $\mathrm{du}$ processus d'autoévaluation critique permettant normalement de moduler le comportement humain (Murphy, 1990). Ces distorsions accroissent la légitimité des gestes faits (p. ex., si un enfant ne dévoile pas les activités sexuelles qu'il a eues avec un adulte, c'est qu'il les apprécie et désire qu'elles se poursuivent), leur acceptabilité (p. ex., si un enfant ne résiste pas, c'est qu'il désire s'engager dans une relation sexuelle avec un adulte) et soutiennent l'aspect non pathologique de la sexualité entre un adulte et un enfant (p. ex., les relations sexuelles sont une bonne façon d'éduquer sexuellement un enfant) (Abel et al., 1984; Bumby, 1996). Les croyances et les attitudes erronées à l'égard des agressions sexuelles constituent des facteurs de risque autant dans l'étiologie que dans le maintien des infractions sexuelles (Abel et al., 1984; Blumenthal, Gudjonsson et Burns, 1999; Burn et Brown, 2006; Moulden, 2008) et augmentent le risque de récidive (Gannon, Ward, Beech et Fisher, 2007; Helmus et al., 2013; Segal et Stermac, 1990). Dans la présente étude, cinq distorsions cognitives sont considérées: les distorsions (1) à l'égard des contacts sexuels entre un adulte et un enfant; (2) à l'égard du viol; (3) traitant du droit à la sexualité; (4) relativement à l'enfant sexualisé; et celles (5) à propos du tort causé par la victimisation sexuelle.

Plusieurs études montrent que les agresseurs sexuels d'enfants adhèrent davantage à des croyances erronées à propos des contacts sexuels entre un adulte et un enfant que des hommes n'ayant pas perpétré de telles infractions (Abel et al., 1989; Bumby, 1996; Marshall, Hamilton et Fernandez, 2001; Moulden, 2008) et particulièrement ceux à haut risque de récidive (Mann, Webster, Wakeling et Marshall, 2007). Bien que ces distorsions cognitives soient centrales chez les 
agresseurs sexuels d'enfants, les distorsions à l'égard du viol semblent également jouer un rôle important. Blumenthal et al. (1999) ont observé que les agresseurs sexuels d'enfants et les agresseurs sexuels d'adultes sont similaires sur le plan des distorsions à l'égard du viol. D'autres auteurs (Arkowitz et Vess, 2003; Olver, Kingston, Nicholaichuk et Wong, 2014) ont même observé davantage de distorsions à l'égard du viol chez les agresseurs sexuels d'enfants comparativement aux agresseurs d'adultes. La notion du droit à la sexualité réfère à la croyance que l'individu a «tous les droits» à propos de la sexualité et qu'il est justifié de combler ses besoins sexuels (Hanson, Gizzarelli et Scott, 1994). Les résultats d'études indiquent que les agresseurs sexuels d'enfants adhèrent davantage aux associations impliquant droit/pouvoir et sexualité que les non-délinquants (Mihailides, Devilly et Ward, 2004; Phelan, 1995). Les distorsions relativement à l'enfant sexualisé représentent la tendance à percevoir les enfants comme sexuellement attirants et désirant avoir une vie sexuelle (Hanson et al., 1994). Les individus perpétrant des infractions sexuelles tendent ainsi à rapporter que les enfants sont sexuellement provocateurs, que leur passivité constitue un accord implicite à participer aux relations sexuelles et qu'ils y prennent plaisir (Abel et al., 1984; Mann et al., 2007; Marziano, Ward, Beech et Pattison, 2006). Enfin, les distorsions quant au tort causé par la victimisation sexuelle réfèrent à la croyance que les gestes sexuels commis ne causent pas ou peu de tort à l'enfant (Hanson et al., 1994). Certains délinquants sexuels rapportent effectivement croire que la sexualité entre un adulte et un enfant est consensuelle (Hanson et al., 1994; Howitt et Sheldon, 2007; Ward et Keenan, 1999) et n'est pas dommageable pour ce dernier (Mann et al., 2007).

\section{Personnalité}

Bien que la documentation scientifique souligne davantage le rôle des distorsions cognitives souvent relevées chez les agresseurs sexuels d'enfants, un nombre plus restreint d'études semble indiquer que certains traits de personnalité sont associés à l'étiologie et au maintien des comportements sexuels déviants (Craig et al., 2007; Hanson et Harris, 2000), à l'adhérence à un traitement (Miner et Dwyer, 1995), ainsi qu'au risque de récidive (Boccaccini, Murrie, Hawes, Simpler et Johnson, 2010 ; Listwan, Van Voorhis et Ritchey, 2007). Par exemple, des études montrent que les hommes accusés d'agression sexuelle à l'égard d'en- 
fants sont susceptibles de présenter un trouble de la personnalité, notamment des traits antisociaux (Bogaerts, Daalder, Vanheule, Desmet et Leeuw, 2008; Cohen et al., 2002), et qu'un diagnostic de trouble de la personnalité augmente de dix fois le risque de récidive (Långström, Sjöstedt et Grann, 2004).

Outre les troubles cliniques francs, les traits de personnalité dits «normaux» peuvent s'avérer utiles afin de prédire les comportements et états affectifs susceptibles d'influencer la réhabilitation et le risque de récidive (Carvalho et Nobre, 2013). Le modèle de la personnalité en cinq facteurs (Costa et McCrae, 1992; Goldberg, 1990) offre un cadre conceptuel bien validé qui permet de décrire la personnalité d'un individu selon cinq dimensions (p. ex., névrosisme, extraversion, ouverture à l'expérience, agréabilité et propension à être consciencieux). Le névrosisme réfère à la propension à ressentir des affects négatifs tels que la peur, la colère et la honte. Les individus présentant un névrosisme élevé ont moins de contrôle sur leurs émotions et réagissent plus fortement au stress (Costa et McCrae, 1992). L'extraversion traduit un besoin d'être entouré, une tendance à l'affirmation de soi, une recherche de sensations et une propension aux émotions positives. Les individus introvertis sont pour leur part réservés et préfèrent la solitude à la présence dans un groupe. L'ouverture caractérise les individus curieux et enclins à adopter des valeurs et des opinions non conventionnelles. À l'inverse, la fermeture traduit une tendance à être conventionnel et à préférer la familiarité. L'agréabilité renvoie à un ensemble de motivations à entretenir des relations positives et à démontrer des comportements prosociaux. L'agréabilité faible réfère, à l'inverse, à l'égocentrisme, au scepticisme et à la compétitivité. Enfin, la propension à être consciencieux implique le respect des normes sociales, le contrôle des impulsions et l'acceptation d'un délai avant la gratification. Les individus peu consciencieux sont quant à eux négligents et impulsifs (Costa et McCrae, 1992).

Madsen, Parsons et Grubin (2006) ont examiné la relation entre les dimensions du modèle en cinq facteurs et les troubles de personnalité du DSM-IV chez un échantillon d'agresseurs sexuels d'enfants. Ils ont observé de fortes associations entre les troubles de la personnalité, le névrosisme et l'antagonisme (faible agréabilité). De plus, comparativement à des groupes contrôles, les agresseurs sexuels présenteraient des niveaux plus élevés de névrosisme et des niveaux plus faibles d'extraversion et de propension à être consciencieux (Becerra-García et al., 
2013; Dennison, Stough et Birgden, 2001; Randall, Carr, Dooley et Rooney, 2011). Certains chercheurs soulignent cependant que, comparativement aux agresseurs sexuels d'adultes, les agresseurs sexuels d'enfants présentent des degrés d'agréabilité et de propension à être consciencieux plus élevés et une ouverture à l'expérience plus faible (Carvalho et Nobre, 2013; Egan, Kavanagh et Blair, 2005), alors que d'autres (Becerra-García, García-León et Egan, 2012) n’indiquent aucune différence entre ces deux groupes. L'absence de distinction entre les agresseurs pédophiles et non pédophiles dans ces échantillons pourrait expliquer les divergences de résultats, en particulier sur le plan de l'agréabilité. En effet, les agresseurs sexuels non pédophiles montrent des niveaux de psychopathie plus élevés que les pédophiles, ces derniers étant davantage motivés par un intérêt sexuel plus exclusif envers l'enfant (Seto, 2007; Strassberg, Eastvold, Wilson Kenney et Suchy, 2012).

Enfin, Becerra-García et al. (2013) concluent que les agresseurs ayant eux-mêmes été victimes d'abus sexuel, physique ou émotionnel dans l'enfance affichent un névrosisme et une ouverture à l'expérience plus élevés, soulignant l'importance d'évaluer l'historique de victimisation.

\section{Programme d'évaluation et de traitement des agressions sexuelles (PÉTAS)}

Depuis 1988, le PÉTAS du Centre jeunesse de la Mauricie et du Centredu-Québec offre des services de psychothérapie aux personnes impliquées dans une situation d'agression sexuelle intrafamiliale. La clientèle du programme touchée par la problématique fait référence à des familles dont un ou des enfants ont été agressés par un parent, un beau-parent, un membre de la fratrie, un membre de la famille élargie (grand-parent, oncle, etc.) ou encore une figure parentale de substitution qui joue un rôle significatif dans la vie de l'enfant. L'agresseur représente le patient identifié et il le demeure tout au long du processus d'intervention individuel, conjugal et familial. Pour plusieurs agresseurs, le traitement est offert sur une base volontaire, une entente étant prise avec l'intervenant au dossier pour diminuer une peine de prison ou accélérer les mesures de transition. Le PÉTAS considère l'agir déviant comme le résultat d'une série d'actes conscients et planifiés, et ce, quoi qu'en disent la plupart des agresseurs au début du traitement. Il comporte une phase d'évaluation (entrevues individuelles et de couple, passation de ques- 
tionnaires), suivie d'un traitement où la thérapie de groupe constitue la stratégie d'intervention privilégiée. Les rencontres de deux heures, au nombre de 54, s'échelonnent sur deux ans. La psychothérapie en groupe se déroule selon quatre phases successives liées à des cibles d'intervention distinctes: 1) orientation (p. ex., reconnaissance de sa responsabilité et du besoin de traitement); 2) sensibilisation voilée (basée sur une technique cognitive-comportementale de type aversif ayant pour but d'associer des stimuli aversifs aux fantaisies déviantes afin de diminuer l'excitation sexuelle associée et d'augmenter le contrôle cognitif des pensées et fantaisies déviantes via la mise à jour des distorsions cognitives; Abel et Rouleau, 1990; Marshall et Barbaree, 1988; Priest et Smith, 1992); 3) prévention de la récidive (p. ex., détermination des aspects de la motivation ayant favorisé l'agir ou l'excitation sexuelle déviante, des besoins sexuels, affectifs, de contrôle et de pouvoir, ainsi que des difficultés à régler adéquatement les conflits; reconnaissance des signes précurseurs d'une agression et élaboration d'un plan de prévention de la récidive); et 4) communication (p. ex., apprentissage des habiletés de communication conjugale et d'une saine gestion de la sexualité; développement de relations interpersonnelles significatives avec des adultes et accroissement de l'intimité et de l'interdépendance conjugale; Wright et Sabourin, 1995).

\section{Objectif}

La présente étude a pour objectif d'examiner les changements sur le plan des distorsions cognitives et de la personnalité à la suite d'un traitement au PÉTAS chez un échantillon d'agresseurs sexuels d'enfants. Il est attendu que l'implication au PÉTAS mène à des diminutions significatives des scores sur l'ensemble des distorsions cognitives et sur le score de névrosisme, ainsi qu'à une augmentation des scores d'extraversion, d'ouverture à l'expérience, d'agréabilité et de propension à être consciencieux.

\section{Méthode}

\section{Participants et procédure}

L'échantillon est composé de 134 hommes en traitement pour agression sexuelle envers un enfant, recrutés à leur entrée au PÉTAS. Dans le cadre du processus d'évaluation (T1), ceux-ci sont invités à remplir une 
batterie de questionnaires incluant, entre autres, des mesures de distorsions cognitives et de la personnalité ainsi qu'un formulaire de consentement à la recherche. Les participants sont invités à remplir à nouveau les questionnaires après la première $(\mathrm{T} 2, n=61)$ et la deuxième année de traitement (T3, $n=37)$. Le projet de recherche a été approuvé par le comité d'éthique de l'Université du Québec à Trois-Rivières.

Les taux d'attrition s'élèvent à $54,4 \%$ au T2 et à $72,4 \%$ au T3. L'abandon du traitement est un problème omniprésent chez cette population, avec des taux variant de $38 \%$ (Browne, Foreman et Middleton, 1998) à $77 \%$ (Hunter Jr et Figueredo, 1999) selon la documentation scientifique. L'attrition peut s'expliquer par plusieurs facteurs, dont la santé mentale, l'abus de substance, le score aux outils actuariels de risque de récidive, le déni du délit commis, la détention en milieu carcéral, etc. (Browne et al., 1998; Craissati et Beech, 2001 ; Hunter Jr et Figueredo, 1999; Nunes et Cortoni, 2008).

$\mathrm{Au} \mathrm{T} 1$, les participants sont âgés de 17 à 72 ans $(M=40,50, E ́ T=$ $14,12)$, ont en moyenne 10,52 années de scolarité $(E ́ T=2,94)$ et gagnent un revenu annuel moyen de $22390 \$(E ́ T=14$ 845,09). Parmi eux, $17,7 \%(n=23)$ sont mariés, $27,7 \%(n=36)$ sont en union de fait, $35,4 \%(n=46)$ sont séparés ou divorcés et ne vivent pas une relation de couple, alors que 19,2\% $(n=25)$ sont séparés ou divorcés et vivent une relation de couple. Aucune différence sur le plan des variables sociodémographiques n'est observée entre les hommes ayant terminé la première année de traitement et ceux ne l'ayant pas terminée, à l'exception du revenu $(t(129)=2,193, p=, 03, d=0,39)$. Les individus ayant terminé la première année de traitement rapportent un revenu plus élevé $(M=25457, E ́ T=16952)$ que ceux ayant abandonné avant la fin de l'année $(M=197163$, É $T=12$ 235).

\section{Instruments}

\section{Distorsions cognitives}

Les distorsions cognitives sont mesurées à l'aide de deux instruments. Le premier questionnaire, le MOLEST AND RAPE Scales (Bumby, 1996), comporte deux sous-échelles. L'échelle MOLEST (37 items) évalue les croyances soutenant la légitimité et l'acceptabilité des contacts sexuels entre un adulte et un enfant, alors que l'échelle RAPE (36 items) évalue celles supportant le viol. Les items sont cotés selon une échelle de type Likert en quatre points $(1=$ Fortement en désaccord, $4=$ Fortement en 
accord). Les réponses aux items d'une sous-échelle sont additionnées, de sorte qu'un résultat élevé indique que le participant utilise davantage de distorsions cognitives. Les échelles MOLEST et RAPE présentent toutes deux des alphas de Cronbach de 0,97 (Bumby, 1996). Au sein de la présente étude, les coefficients sont respectivement de 0,85 et 0,88.

En deuxième lieu, trois échelles du Hanson Sex Attitudes Questionnaire sont retenues (Hanson et al., 1994): les échelles de l'enfant sexualisé (11 items), du droit à la sexualité (9 items) et du tort causé par la victimisation sexuelle (10 items). Une échelle de type Likert en cinq points est utilisée $(1=$ Complètement en désaccord, $5=$ Complètement en accord $)$. Les réponses aux items sont additionnées, un résultat élevé à une souséchelle indiquant que le participant utilise davantage ce type de distorsions cognitives. Les coefficients de cohérence interne pour les trois échelles sont supérieurs à 0,80 (Hanson et al., 1994). Au sein de la présente étude, les coefficients sont de 0,85 pour l'échelle de l'enfant sexualisé, de 0,63 pour l'échelle du droit à la sexualité et de 0,71 pour l'échelle du tort causé par la victimisation sexuelle.

\section{Personnalité}

Les traits de personnalité sont mesurés à l'aide du NEO-Five Factor Inventory (NEO-FFI; Costa et McCrae, 1992). Ce questionnaire autorapporté évalue les dimensions du modèle en cinq facteurs. Chaque sous-échelle comprend 12 items auxquels répondre sur une échelle de type Likert en cinq points, évaluant dans quelle mesure l'individu considère l'énoncé comme étant représentatif de lui-même $(1=$ Fortement en désaccord, $5=$ Fortement en accord). Les items pour chaque sous-échelle sont additionnés, un score élevé représentant un niveau élevé du trait. Les scores bruts sont ensuite convertis en scores $\mathrm{T}$ à partir d'un échantillon de normalisation. Les étendues suivantes sont suggérées (Costa et McCrae, 1992) pour interpréter les scores: faible (score $\mathrm{T}<45$ ), modéré (score $\mathrm{T}=45-55$ ) et élevé (score $\mathrm{T}>55$ ). La version française du questionnaire a été développée par Sabourin et Lussier (1992) et présente une validité de construit et une fidélité adéquates. Au sein de la présente étude, les coefficients alpha varient de 0,57 à 0,82 .

\section{Historique de victimisation en enfance}

Afin de vérifier la présence d'agressions sexuelles subies, la question suivante est utilisée: "Avez-vous déjà été abusé sexuellement pendant votre enfance et/ou adolescence?» L'item est coté de façon dichotomique 
$(1=$ Oui, $0=$ Non $)$. Quatre items examinent la fréquence à laquelle l'individu a été victime ou témoin de violence psychologique et physique de la part des parents. Chaque item varie de 0 à $4(0=$ Jamais, $4=$ Très souvent). Les items sont ensuite additionnés afin de créer un score continu représentant la sévérité de l'exposition à la violence familiale.

\section{Caractéristiques des agressions perpétrées}

Diverses questions sont utilisées afin de documenter la nature des agressions perpétrées. L'item «Nombre d'enfants que vous avez abusés sexuellement» est dichotomisé afin de discriminer les individus rapportant une victime de ceux rapportant plusieurs victimes. Le type d'acte commis est évalué afin de créer une variable représentant la sévérité de l'agression perpétrée. Pour ce faire, les différents actes sont regroupés selon trois catégories: «sans contact» (p. ex., propositions verbales pour des actes sexuels, obligation de regarder des scènes sexuelles, voyeurisme ou exhibitionnisme), «avec attouchements» (p. ex., attouchements sexuels [oraux ou avec les mains] faits ou reçus), et «avec pénétration» (p. ex., relations sexuelles complètes). L'acte le plus sévère se voit attribuer un score, allant de $1=$ sans contact à $3=$ avec pénétration. Enfin, la question «Avez-vous déjà purgé une peine de prison?» est utilisée afin d'évaluer l'historique d'incarcération.

\section{Résultats}

\section{Analyses descriptives}

Les statistiques descriptives pour les distorsions cognitives et les traits de personnalité au moment de l'évaluation (T1) sont présentées dans le Tableau 1. De 10 à $50 \%$ des hommes affichent des scores cliniquement élevés selon les différentes dimensions de la personnalité, ce qui indique que 50 à $90 \%$ ne présentent pas de scores problématiques à leur entrée au PÉTAS. L'absence de point de coupure validé sur les échelles de distorsions ne permet pas de documenter la présence de scores cliniquement significatifs sur ces variables.

Les prévalences pour différents types de victimisation en enfance au sein de l'échantillon sont présentées au Tableau 1. Les hommes rapportant avoir été victimes d'agression sexuelle montrent des degrés d'agréabilité significativement plus faibles au T1 $(M=48,09, E ́ T=12,35)$ que ceux ne rapportant pas d'agression sexuelle subie $(M=52,86$, 
$\dot{E} T=10,72), t(132)=2,39, p=0,018, d=0,42^{2}$. Des associations significatives sont également observées entre l'intensité de l'exposition à la violence familiale et le névrosisme, $r(132)=0,27, p=0,002$, l'extraversion, $r(132)=-0,20, p=0,02$ et l'agréabilité, $r(132)=-0,32$, $p<, 001$.

Les caractéristiques des agressions perpétrées sont présentées dans le Tableau 1. Comparativement aux hommes rapportant des agressions sur une victime, ceux rapportant plusieurs victimes montrent des niveaux significativement plus élevés de névrosisme $\left(M_{1}=60,50, E ́ T_{1}\right.$ $\left.=11,96 ; M_{2}=54,02, E T_{2}=11,26\right) t(131)=-2,95, p=0,004, d=$ 0,56 , ainsi que des taux plus faibles d'extraversion $\left(M_{1}=49,11, E ́ T_{1}=\right.$ 9,36; $\left.M_{2}=54,96, E ́ T_{2}=9,59\right) t(131)=3,20, p=0,002, d=0,62$ et d'agréabilité $\left(M_{1}=46,97, E ́ T_{1}=13,61 ; M_{2}=52,33, E ́ T_{2}=10,51\right) t(131)$ $=2,43, p=0,016, d=0,44$. De plus, les hommes ayant été incarcérés présentent des degrés d'agréabilité significativement plus faibles $(M=46,71, E ́ T=13,81)$ que ceux n'ayant pas été incarcérés $(M=52,28$, $\hat{E} T=10,46), t(48,51)=2,48, p=0,035, d=0,46$. La sévérité de l'agression n'est associée à aucune des variables à l'étude. De plus, l'historique de victimisation et les caractéristiques des agressions ne permettent pas de prédire des différences sur le plan des distorsions cognitives.

\section{Attrition}

Étant donné le taux important d'attrition entre les différents temps de mesure, les hommes ayant quitté prématurément sont comparés à ceux ayant terminé le traitement sur le plan des distorsions cognitives et de la personnalité. Les résultats d'analyses de variance (ANOVA) à un facteur semblent indiquer une seule différence significative: les individus ayant abandonné le traitement présentent une ouverture à l'expérience significativement plus faible au T1 $(M=42,71, E ́ T=13,19)$ que ceux ayant terminé la première année de traitement $(M=47,39$, $\hat{E} T=11,79), F(1,132)=4,61, p=0,03, d=0,37$. Le pourcentage d'individus présentant des scores de personnalité cliniquement significatifs est également examiné. Des résultats similaires sont observés, indiquant que les individus ayant abandonné le traitement sont signi-

2. L'amplitude des tailles d'effet s'interprète comme suit: $d=0,2$ est considérée faible, $d=0,5$ est considérée moyenne et $d=0,8$ est considérée forte (Cohen, 1992). 
TABLEA U 1

Statistiques descriptives pour différentes variables à l'entrée au PÉTAS $(N=134)$

\begin{tabular}{|c|c|c|c|c|c|}
\hline Variables & $M$ & $\bar{E} T$ & Étendue & $\%$ & $n$ \\
\hline \multicolumn{6}{|l|}{ Distorsions cognitives } \\
\hline Agression sexuelle d'enfants & 62,91 & 14,83 & $37-101$ & & \\
\hline Viol & 57,70 & 15,06 & $36-112$ & & \\
\hline Enfant sexualisé & 17,44 & 7,13 & $11-38$ & & \\
\hline Droit à la sexualité & 18,75 & 5,45 & $9-37$ & & \\
\hline Tort causé par les agressions & 14,29 & 4,45 & $10-32$ & & \\
\hline \multicolumn{6}{|l|}{ Personnalitéa } \\
\hline Névrosisme & 55,87 & 11,74 & $26-86$ & 50,0 & 67 \\
\hline Extraversion & 53,21 & 9,86 & $29-74$ & 18,7 & 25 \\
\hline Ouverture à l'expérience & 44,84 & 12,74 & $17-76$ & 44,8 & 60 \\
\hline Agréabilité & 50,83 & 11,64 & $16-77$ & 25,4 & 34 \\
\hline Propension à être consciencieux & 56,67 & 10,02 & $20-73$ & 10,4 & 14 \\
\hline \multicolumn{6}{|l|}{ Victimisation en enfance (prévalence) } \\
\hline Agression sexuelle & & & & 42,5 & 57 \\
\hline Témoin de violence psychologique & & & & 47,8 & 64 \\
\hline Témoin de violence physique & & & & 20,9 & 28 \\
\hline Victime de violence psychologique & & & & 46,3 & 62 \\
\hline Victime de violence physique & & & & 42,5 & 57 \\
\hline \multicolumn{6}{|l|}{ Agression perpétrée la plus sévère } \\
\hline Sans contact & & & & 0,8 & 1 \\
\hline Attouchements & & & & 86,4 & 114 \\
\hline Avec pénétration & & & & 12,9 & 17 \\
\hline \multicolumn{6}{|l|}{ Lien avec la victime } \\
\hline Père & & & & 30,6 & 41 \\
\hline Conjoint de la mère & & & & 22,4 & 30 \\
\hline Tuteur légal & & & & 0,7 & 1 \\
\hline Oncle & & & & 6,0 & 8 \\
\hline Grand-père & & & & 6,0 & 8 \\
\hline Cousin & & & & 14,2 & 19 \\
\hline Frère & & & & 26,1 & 35 \\
\hline Nombre de victimes ${ }^{b}$ & 1,59 & 1,23 & $1-7$ & 28,6 & 38 \\
\hline Incarcération & & & & 26,1 & 35 \\
\hline
\end{tabular}

Notes: $\mathrm{a}=$ Les pourcentages reflètent la proportion d'individus se situant dans l'étendue clinique selon les critères de Costa et McCrae, 1992 (Névrosisme > 55 et extraversion, ouverture, agréabilité et propension à être consciencieux $<45) ; b=$ Le pourcentage indique la proportion d'individus ayant perpétré des agressions sur plus d'une victime. 
ficativement plus nombreux à présenter des scores d'ouverture cliniquement faibles $(52,1 \%, n=38)$ au T1 que les individus ayant complété la première année de traitement $(36,1 \%, n=22), \chi^{2}(1)=3,44, p=0,05$. Aucune autre différence n'est observée pour les autres traits de personnalité. La possibilité que le biais d'attrition contribue au changement pré- et post-traitement semble donc fortement diminuée.

\section{Évolution des distorsions cognitives et de la personnalité}

Afin d'évaluer les changements sur le plan des distorsions cognitives et de la personnalité après la première et la deuxième année de traitement, différentes stratégies sont utilisées. Premièrement, des ANOVA à mesures répétées sont effectuées. Deuxièmement, l'indice de fiabilité du changement (Jacobson, Follette et Revenstorf, 1984) est calculé afin d'examiner la proportion d'individus présentant un changement significatif sur chacune des mesures. Une attention particulière est portée aux individus présentant des scores problématiques au prétraitement (T1).

\section{Analyses de variances à mesures répétées}

Étant donné le taux important d'attrition entre les différents temps de mesure, les analyses ont été effectuées à l'aide du logiciel Mplus (Muthén et Muthén, 1998-2016). Mplus permet la gestion des données manquantes selon la méthode Full Information Maximum Likelihood. Le taux d'attrition supérieur à $50 \%$ entre le T1 et le T2 rend impossible la gestion des données manquantes entre ces deux temps de mesure. Par contre, le taux d'attrition moindre (autour de $40 \%$ ) entre les T2 et T3 le permet, avec un échantillon de 61 participants. Le Tableau 2 présente les résultats des ANOVA à mesures répétées pour chacune des variables examinées.

Des diminutions significatives sont observées sur le nombre de distorsions à l'égard des contacts sexuels entre un enfant et un adulte. Les comparaisons à postériori (avec correction de Bonferroni) supposent une diminution entre les T1 et T2, Wald $=53,05, p<0,001$, entre les T1 et T3, Wald $=87,34, p<0,001$, et entre les T2 et T3, Wald $=13,21$, $p<0,001$. Des diminutions significatives sont également observées sur le plan du nombre de distorsions sur le viol entre les T1 et T2, Wald = 33,59, $p<0,001$, et entre les T1 et T3, Wald $=40,89, p<0,001$. Une diminution des croyances quant à l'enfant sexualisé est observée entre les $\mathrm{T} 1$ et $\mathrm{T} 2$, Wald $=10,59, p=0,001$, et une diminution des croyances sur 


\section{TA B LEAU 2}

Résultats des ANOVA à mesures répétées pour les distorsions cognitives et les dimensions de la personnalité $(n=61)$

\begin{tabular}{|c|c|c|c|c|c|c|c|c|}
\hline \multirow[b]{2}{*}{ Variables } & & \multicolumn{2}{|c|}{ T1 } & \multicolumn{2}{|c|}{$\mathrm{T} 2$} & \multicolumn{2}{|c|}{ T3 } & \multirow[b]{2}{*}{ Wald } \\
\hline & & $M$ & $\bar{E} T$ & $M$ & $\hat{E} T$ & $M$ & $\dot{E} T$ & \\
\hline \multirow[t]{5}{*}{ Distorsions cognitives } & AS d'enfants & $63,72^{\mathrm{a}}$ & 14,84 & $53,03^{b}$ & 14,52 & $48,52^{c}$ & 12,16 & $86,06 * * *$ \\
\hline & Viol & $57,54^{\mathrm{a}}$ & 15,51 & $49,93^{b}$ & 14,48 & $47,38^{b}$ & 12,97 & $44,62 * * *$ \\
\hline & Enfant sex & $14,48^{\mathrm{a}}$ & 7,41 & $14,85^{b}$ & 5,73 & $15,12^{\mathrm{a}, \mathrm{b}}$ & 6,58 & $10,67^{* *}$ \\
\hline & Droit sex & $19,13^{\mathrm{a}}$ & 5,85 & $17,10^{\mathrm{b}}$ & 5,61 & $16,82^{b}$ & 5,44 & 10,90 ** \\
\hline & Tort & $14,39^{\mathrm{a}}$ & 4,53 & $12,87^{b}$ & 3,95 & $13,02^{a, b}$ & 3,82 & $6,04^{*}$ \\
\hline \multirow[t]{5}{*}{ Personnalité } & Névrosisme & $56,87^{\mathrm{a}}$ & 10,72 & $53,93^{a}$ & 11,56 & $50,66^{b}$ & 9,33 & $19,25^{* * *}$ \\
\hline & Extraversion & $53,74^{\mathrm{a}}$ & 9,63 & $56,03^{a, b}$ & 8,82 & $58,67^{b}$ & 7,93 & $14,83^{* * *}$ \\
\hline & Ouverture & $47,39^{a}$ & 11,70 & $48,64^{a}$ & 12,12 & $50,59^{a}$ & 10,44 & 4,98 \\
\hline & Agréabilité & $50,28^{a}$ & 11,59 & $51,69^{\mathrm{a}}$ & 11,12 & $50,53^{\mathrm{a}}$ & 10,89 & 1,27 \\
\hline & Conscience & $58,16^{\mathrm{a}}$ & 9,22 & $59,31^{\mathrm{a}}$ & 8,16 & $58,51^{\mathrm{a}}$ & 7,68 & 1,57 \\
\hline
\end{tabular}

Notes: $\mathrm{AS}=$ agression sexuelle; Enfant sex = enfant sexualisé; Droit sex = droit à la sexualité; Tort = tort causé par la victimisation sexuelle; Conscience = propension à être consciencieux. Les moyennes accompagnées de lettres différentes diffèrent significativement $(p<, 05) .{ }^{*} p<, 05 .{ }^{* *} p<, 01 .{ }^{* *} p<, 001$. 
le droit à la sexualité est notée entre les T1 et T2, Wald $=8,78, p=0,003$, et les T1 et T3, Wald $=8,98, p=0,002$. Finalement une diminution des croyances quant au tort lié à la victimisation est observée entre les T1 et T2, Wald $=6,02, p=0,014$. Sur le plan de la personnalité, une diminution significative est observée pour le névrosisme. Les comparaisons à postériori avec correction de Bonferroni montrent une diminution entre le T1 et le T3, Wald $=19,24, p<0,001$, ainsi qu'entre le T2 et le T3, $W$ ald $=6,19, p=0,013$. Les résultats semblent également indiquer une augmentation de l'extraversion entre les T1 et T3, Wald $=14,78, p<$ 0,001 .

Indice de fiabilité du changement

L'Indice de fiabilité du changement (The Reliability of Change Index; Christensen et Mendoza, 1986; Jacobson et al., 1984), appliqué dans diverses études de traitement des agresseurs sexuels (p. ex., Beech et Ford, 2006; Mandeville-Norden, Beech et Hayes, 2008; Nunes, Babchishin et Cortoni, 2011) constitue une solution de rechange aux ANOVA pour examiner les changements pré- et post-traitement (Mandeville-Norden et al., 2008). Ces dernières, bien qu'elles permettent de déterminer si un changement global est observé au sein de l'échantillon, informent peu sur le changement sur le plan individuel. L'indice de fiabilité du changement (IFC) permet quant à lui de documenter la présence de changement sur une variable donnée pour chaque individu de l'échantillon, en tenant compte du degré de fiabilité de l'instrument. Cet indice permet donc de distinguer le changement réel des variations dues à l'erreur de mesure (Robins, Fraley, Roberts et Trzesniewski, 2001). Il se traduit par une taille d'effet significative à $p=0,05$ (unilatéral) lorsque sa valeur est égale ou supérieure à 1,64 (ou inférieure à -1,64 dans le cas d'une diminution significative). Cette méthode permet également de documenter le nombre d'individus s'étant rétablis, c'est-à-dire ceux montrant à la fois une amélioration significative et un score se situant dans la distribution de scores des individus fonctionnels. Pour chaque mesure, le point de coupure à partir duquel un score est considéré comme faisant partie de la distribution des scores des individus fonctionnels est calculé selon la procédure proposée par Jacobson et Truax (1991) ${ }^{3}$. La proportion d'individus

3. Pour les traits de personnalité, la distribution des scores des individus fonctionnels (selon les normes du NEO-FFI; Costa et McCrae, 1992) et la distribution des scores des 
ne présentant aucun changement (taille d'effet $<1,64$ ) et celle des individus s'étant détériorés (taille d'effet $>1,64$ dans la direction opposée à celle attendue) peuvent également être documentées. Cet indice est utilisé pour examiner la proportion d'individus dans chacune de ces quatre catégories, sur le plan des distorsions (diminution du score) et des traits de personnalité (diminution du névrosisme et augmentation de l'extraversion, de l'ouverture, de l'agréabilité et de la propension à être consciencieux) après la première année (T2) et après la deuxième année de traitement (T3). Les résultats sont présentés dans le Tableau 3.

En ce qui a trait aux hommes montrant une amélioration significative (incluant ceux étant rétablis) les proportions varient grandement selon les variables, allant de 16 à $39 \%(M d n=19,30)$ après la première année et de 14 à $54 \%(M d n=27,82)$ après la deuxième année. Étant donné qu'un changement sur le plan de la personnalité est particulièrement souhaitable pour les individus présentant des scores dans l'étendue clinique au T1, l'IFC est également examiné spécifiquement parmi ceux-ci. Les résultats, présentés au Tableau 3, montrent que le traitement semble amener davantage de changements chez les individus qui présentent des scores cliniquement élevés à leur entrée au PÉTAS. Après un an de traitement, environ le tiers de ces participants montrent soit des améliorations ou sont rétablis sur le plan du névrosisme, de l'ouverture et de l'agréabilité, alors que la moitié des participants montrent des améliorations ou sont rétablis sur le plan de l'extraversion et de la propension à être consciencieux. Les changements tendent à augmenter après deux années de traitement, avec plus de $70 \%$ des hommes présentant des changements significatifs ou étant rétablis sur le plan du névrosisme, de l'extraversion et de la propension à être consciencieux.

En ce qui a trait aux distorsions cognitives, les améliorations les plus importantes sont observées pour les distorsions quant aux agressions sexuelles à l'égard d'enfants et celles à l'égard du viol (voir Tableau 3). Par exemple, 39,3\% des agresseurs affichent des changements significatifs sur le plan de leurs distorsions à l'égard de l'agression sexuelle d'enfants, et ce pourcentage atteint $54,1 \%$ après deux ans de traitement.

participants à l'étude se chevauchent. L'équation suivante est donc utilisée afin de dériver les points de coupure: $S_{0} M_{1}+S_{1} M_{0} / S_{0}+S_{1}$ Pour ce qui est des distorsions cognitives, aucune information n'est disponible quant à la distribution des scores des individus fonctionnels. Le point de coupure est donc situé à deux écart-types au-dessous de la moyenne. 
TABLEA U 3

Proportion d'hommes présentant une amélioration significative, rétablis, ne présentant aucun changement ou s'étant détériorés sur le plan de la personnalité et des distorsions cognitives à la suite du traitement

\begin{tabular}{|l|c|c|c|c|c|c|c|c|}
\hline & \multicolumn{9}{|c|}{ Proportion d'hommes dans chaque catégorie } \\
\hline & \multicolumn{7}{|c|}{ Échantillon total } & \multicolumn{3}{c|}{ Parmi ceux dans l'étendue clinique au T1 } \\
\hline & $\begin{array}{c}\text { Amélioration } \\
\text { significative }\end{array}$ & Rétablis & $\begin{array}{c}\text { Aucun } \\
\text { changement }\end{array}$ & Détériorés & $\begin{array}{c}\text { Amélioration } \\
\text { significative }\end{array}$ & Rétablis & $\begin{array}{c}\text { Aucun } \\
\text { changement }\end{array}$ & \begin{tabular}{c} 
Détériorés \\
\hline
\end{tabular} \\
\hline
\end{tabular}


TABLEAU 3 (SUITE)

\begin{tabular}{|c|c|c|c|c|c|c|c|c|}
\hline \multirow[b]{3}{*}{ Distorsions cognitives } & \multicolumn{4}{|c|}{ Échantillon total } & \multirow{3}{*}{$\begin{array}{c}M_{\mathrm{T} 1} \mathrm{gr} \text {. avec } \\
\text { amélioration }\end{array}$} & \multirow{3}{*}{$\begin{array}{c}M_{\mathrm{T} 1} \text { gr. sans } \\
\text { amélioration }\end{array}$} & \multirow[b]{3}{*}{$t$} & \multirow[b]{3}{*}{$d$} \\
\hline & \multirow{2}{*}{\begin{tabular}{|c}
$\begin{array}{c}\text { Amélioration } \\
\text { significative }\end{array}$ \\
$\%(n)$
\end{tabular}} & \multirow{2}{*}{$\begin{array}{l}\text { Rétablis } \\
\%(n)\end{array}$} & \multirow{2}{*}{$\begin{array}{c}\begin{array}{c}\text { Aucun } \\
\text { changement }\end{array} \\
\%(n)\end{array}$} & \multirow{2}{*}{$\begin{array}{l}\text { Détériorés } \\
\%(n)\end{array}$} & & & & \\
\hline & & & & & & & & \\
\hline \multicolumn{9}{|l|}{ Après un an de traitement } \\
\hline AS d'enfants & $39,3(24)$ & - & $60,7(37)$ & - & 71,21 & 58,86 & $-3,42^{* *}$ & 0,90 \\
\hline Viol & $24,6(15)$ & - & $72,1(44)$ & $3,3(2)$ & 68,80 & 53,87 & $-3,50^{* *}$ & 1,04 \\
\hline Enfant sex & $18,0(11)$ & - & $75,4(46)$ & $6,6(4)$ & 26,91 & 15,40 & $-5,72^{* * *}$ & 1,91 \\
\hline Droit & $19,7(12)$ & - & $72,1(44)$ & $8,2(5)$ & 23,33 & 18,10 & $-2,92^{* *}$ & 0,94 \\
\hline Tort & $18,0(11)$ & - & $77,0(47)$ & $4,9(3)$ & 20,55 & 13,04 & $-6,35^{* * *}$ & 2,12 \\
\hline \multicolumn{9}{|c|}{ Après deux ans de traitement } \\
\hline AS d'enfants & $54,1(20)$ & - & $43,2(16)$ & $2,7(1)$ & 68,20 & 57,71 & $-2,54^{*}$ & 0,84 \\
\hline Viol & $37,8(14)$ & - & $62,2(23)$ & - & 70,00 & 50,61 & $-4,34^{* * *}$ & 1,47 \\
\hline Enfant sex & $13,5(5)$ & - & $81,1(30)$ & $5,4(2)$ & 29,20 & 15,91 & $-4,24^{* * *}$ & 2,04 \\
\hline Droit & $24,3(9)$ & - & $73,0(27)$ & $2,7(1)$ & 23,22 & 18,82 & $-1,78$ & 0,68 \\
\hline Tort & $18,9(7)$ & - & $73,0(27)$ & $8,1(3)$ & 21,71 & 12,73 & $-6,99 * * *$ & 2,93 \\
\hline
\end{tabular}

Notes: Conscience $=$ propension à être consciencieux; $A S=$ agression sexuelle; Enfant sex $=$ enfant sexualisé Droit $=$ droit à la sexualité Tort $=$ tort lié aux agressions. Les colonnes $《 M$ gr. avec amélioration» et « $M_{\mathrm{T}}$ gr. sans amélioration» présentent les moyennes au T1 pour chacune des distorsions cognitives pour les individus présentant une amélioration significative

en cours de traitement et ceux ne présentant pas d'amélioration significative. * $p<0,05 * * *<<0,01 * * * *<0,001$. 
Étant donné qu'aucun score validé n'existe pour les échelles de distorsions cognitives, des comparaisons de moyennes sont effectuées afin de vérifier si les hommes montrant des changements significatifs au posttraitement présentaient des scores plus problématiques au T1 comparativement à ceux qui ne présentaient pas d'amélioration. Les résultats semblent indiquer qu'à l'exception des croyances quant au droit à la sexualité, les individus présentant des améliorations significatives au post-traitement obtenaient effectivement des scores significativement plus problématiques au prétraitement.

\section{Discussion}

L'objectif de l'étude était d'examiner l'évolution des distorsions cognitives et de la personnalité, deux catégories de variables associées à l'étiologie, au maintien et au risque de récidive de délits sexuels à l'égard d'enfants, à la suite d'un traitement de groupe au PÉTAS. Les analyses effectuées ont indiqué des diminutions significatives sur la totalité des distorsions cognitives ainsi qu'une diminution du névrosisme et une augmentation de l'extraversion chez les participants. Sur le plan personnel, la proportion d'individus présentant des changements significatifs à la suite du traitement varie selon les variables et le nombre d'années de traitement. En général, les résultats indiquent des proportions plus importantes d'hommes montrant des améliorations significatives parmi ceux ayant terminé les deux années de traitement. Un examen attentif permet également de constater que les hommes montrant des changements significatifs à la fin du traitement sont en majorité ceux qui présentaient des scores plus problématiques lors de leur entée au PÉTAS. Il est important de noter qu'en ce qui a trait aux distorsions cognitives, l'absence d'hommes s'étant rétablis selon les critères de Jacobson et al. (1984) pourrait être attribuable au point de coupure particulièrement conservateur de deux écarts types au-dessus de la moyenne. Les résultats semblent tout de même indiquer que les distorsions et les croyances contribuant au maintien d'agressions sexuelles sont remises en question chez une proportion significative d'individus, par les interventions du PÉTAS. L'influence du traitement sur le névrosisme et l'extraversion laisse également entendre que le programme a permis d'augmenter la stabilité émotionnelle et de favoriser l'acquisition de compétences interpersonnelles chez la majorité des hommes qui présentaient des déficits à leur entrée au programme. Chez 
les quelques individus qui faisaient initialement preuve d'une faible propension à être consciencieux, le fait d'avoir terminé le traitement semble avoir favorisé un meilleur contrôle des impulsions. Une réflexion par rapport à l'efficacité limitée du traitement pour augmenter l'ouverture à l'expérience et l'agréabilité apparaît toutefois nécessaire afin de mieux intervenir sur le plan de ces caractéristiques.

\section{Description de l'échantillon à l'entrée au PÉTAS}

En accord avec les hauts taux de victimisation en enfance observés chez les délinquants sexuels (Jespersen, Lalumière et Seto, 2009), les hommes de l'échantillon ont rapporté avoir été victimes de violence psychologique, physique et sexuelle dans une proportion importante. Notamment, la prévalence de la victimisation sexuelle et de l'exposition à la violence conjugale au sein de l'échantillon est quatre fois plus élevée que celle observée chez les hommes de la population générale québécoise (Flores, Laforest et Joubert, 2016; Hébert et al., 2009) et celle de la violence physique, près de deux fois plus élevée (Flores et al., 2016). De plus, au sein de la présente étude, un historique de victimisation est associé à un névrosisme élevé et à une extraversion et à une agréabilité faibles lors de l'évaluation. Cette constellation de traits a spécifiquement été associée à la perpétration d'agressions sexuelles (Becerra-García et al., 2012; Becerra-García et al., 2013; Carvalho et Nobre, 2013; Randall et al., 2011). Étant donné que la majorité des hommes ayant été victimisés dans l'enfance ne deviennent pas eux-mêmes agresseurs, ceux présentant également une constellation de traits de personnalité indésirables pourraient être particulièrement à risque. Les individus présentant un névrosisme élevé sont caractérisés par une forte réactivité aux émotions négatives et une difficulté marquée à réguler leurs affects intenses. La faible extraversion est susceptible de traduire une tendance à l'isolement et des habiletés sociales déficientes. Enfin, la faible agréabilité peut se manifester par de la méfiance et de l'agressivité (Costa et McCrae, 1992). Au sein de la présente étude, ces mêmes traits de personnalité ont également été plus fortement observés chez les hommes rapportant des agressions sexuelles sur plusieurs victimes, comparativement à ceux rapportant une victime. Enfin, des degrés plus faibles d'agréabilité sont observés chez les individus ayant été incarcérés, soulignant la tendance à l'antagonisme et à la manipulation chez ce sous-groupe d'hommes. Ces observations semblent indiquer que l'his- 
torique de victimisation mérite d'être évalué lors de la prise en charge d'individus consultant pour des délits sexuels puisqu'il semble être précurseur d'un profil de personnalité associé au risque de perpétration et de récidive.

\section{Pistes de réflexion pour l'optimisation du traitement}

Les résultats de la présente étude sont fort encourageants quant à la possibilité de modifier certains traits de personnalité dysfonctionnels et distorsions cognitives chez des agresseurs sexuels d'enfants. En se basant sur des résultats méta-analytiques quant à l'efficacité des traitements psychologiques destinés aux délinquants sexuels, Grønnerød, Grønnerød et Grøndahl (2015) concluent que des traitements plus longs et intensifs pourraient s'avérer avantageux. C'est probablement ce qui explique en partie les changements favorables observés à la suite de l'implication au PÉTAS, un traitement en groupe de longue durée ( 2 ans).

Selon Andrews et Bonta (2010), les traitements les plus efficaces seraient ceux pour lesquels l'intensité concorde avec le risque de récidive de l'individu. Ces traitements devraient également cibler des aspects du fonctionnement psychologique, social et émotionnel de l'individu qui sont liés à la poursuite des comportements criminels, tels que les attitudes soutenant ces comportements, les caractéristiques antisociales, les accomplissements sociaux (emploi, degré de scolarité), le fonctionnement conjugal et familial, l'abus de substance, etc. Enfin, ces traitements devraient être individualisés et s'adapter à la personnalité, aux capacités cognitives, d'apprentissage et d'adaptation, à la motivation, ainsi qu'au contexte culturel de chacun. Une attention particulière à ces facteurs pourrait contribuer à augmenter l'efficacité du PÉTAS sur le plan de caractéristiques qui sont pour l'instant moins influencées par le traitement, telles que la faible ouverture et la faible agréabilité.

Enfin, la non-adhérence au traitement est une difficulté couramment rencontrée chez les agresseurs sexuels qui a des conséquences importantes sur le plan du risque de récidive (Olver et Wong, 2011). Au sein de la présente étude, les hommes ayant abandonné prématurément le programme présentaient des degrés plus faibles d'ouverture à l'expérience. Puisque la faible ouverture caractérise une tendance à adopter des opinions plus rigides ainsi qu'une faible propension à s'exposer à de nouvelles expériences et à accepter des perspectives différentes, il 
est probable qu'il soit particulièrement exigeant pour ces individus d'adhérer à un traitement dédié à la confrontation des croyances et des schémas de pensées dysfonctionnels. Cette observation suppose la nécessité de porter une attention particulière au degré d'ouverture de l'individu dans la gestion du risque d'abandon et des obstacles au traitement.

\section{Limites de l'étude}

La présente étude comporte certaines limites nécessitant d'en nuancer les conclusions. Premièrement, l'absence de groupe de comparaison ne permet pas d'examiner les différences entre les individus de la population générale et les hommes de l'échantillon. La disponibilité de scores normalisés pour les variables de personnalité et de prévalences de la victimisation en enfance au sein de la population générale pallie en partie cette limite. Par contre, l'absence de groupe de comparaison ne permet pas d'affirmer avec certitude que les changements observés sont dus au traitement ou au passage du temps. Bien que les variables à l'étude soient considérées comme stables ou montrent des changements sur des périodes de temps beaucoup plus grandes (Roberts, Walton et Viechtbauer, 2006), la réalisation d'études cliniques randomisées demeure la stratégie le plus fiable pour l'étude d'efficacité des traitements (Grønnerød et al., 2015). Le recours à de tels devis de recherche demande cependant des ressources importantes et doit s'appuyer sur des données préliminaires prometteuses. C'est maintenant le cas du PÉTAS. Deuxièmement, le taux d'attrition élevé d'une période d'observation à l'autre limite les conclusions des analyses statistiques. D'un côté, les analyses préliminaires semblent indiquer une influence limitée de l'attrition sur les principaux résultats. Cependant, la diminution de l'échantillon à chacun des temps de mesure crée une perte de puissance statistique réduisant la capacité des analyses à trouver des différences significatives entre le pré- et le post-traitement (Cohen, 1998). Troisièmement, l'absence de point de coupure sur les mesures de distorsions cognitives ne permet pas de documenter la prévalence de scores cliniquement significatifs sur ces variables aux différents temps de mesure. Quatrièmement, l'utilisation d'un item dichotomisé d'historique d'agression sexuelle est susceptible de sous-estimer le nombre d'hommes ayant été victimisés dans l'enfance. En effet, les hommes tendent à sous-rapporter certaines expériences d'agression sexuelle (p. ex., expé- 
rience sexuelle avec une femme plus âgée; Vaillancourt-Morel et al., 2016). De plus, un item dichotomique ne permet pas d'évaluer la sévérité ou la chronicité des abus sexuels subis. Cinquièmement, la présente étude documente les changements sur le plan des distorsions cognitives et de la personnalité, des facteurs associés au risque de récidive. Cependant, les résultats ne permettent pas de conclure directement à une réelle diminution de la récidive. De plus, l'utilisation d'un outil d'évaluation du risque de récidive aurait été nécessaire afin de vérifier si ce facteur explique en partie l'attrition observée au cours du traitement. Ces aspects de l'efficacité du traitement sont centraux et devraient être abordés dans les recherches futures quant à l'efficacité du PÉTAS. Enfin, une prise en compte du diagnostic de pédophilie pourrait permettre un examen plus détaillé des traits de personnalité et des distorsions cognitives chez les agresseurs sexuels pédophiliques et non pédophiliques ainsi que de comparer le risque de récidive et la réponse au traitement chez ces deux sous-groupes de délinquants sexuels.

\section{Références}

Abel, G. G., Becker, J. V. et Cunningham-Rathner, J. (1984). Complications, consent, and cognitions in sex between children and adults. International Journal of Law and Psychiatry, 7, 89-103.

Abel, G. G., Gore, D. K., Holland, C. L., Camp, N., Becker, J. V. et Rathner, J. (1989). The measurement of the cognitive distortions of child molesters. Annals of Sex Research, 2, 135-153.

Abel, G. G. et Rouleau, J.-L. (1990). The nature and extent of sexual assault. Dans W. L. Marshall, D. R. Laws et H. E. Barbaree (dir.), Handbook of sexual assault: Issues, theories, and treatment of the offender (p. 9-21). New York, NY: Plenum Press.

Andrews, D. A et Bonta, J. (2010). Rehabilitating criminal justice policy and practice. Psycbology, Public Policy, and Law, 16(1), 39-55.

Andrews, D. A., Bonta, J. et Wormith, J. S. (2006). The Recent Past and Near Future of Risk and/or Need Assessment. Crime \& Delinquency, 52(1), 7-27.

Arkowitz, S. et Vess, J. (2003). An Evaluation of the Bumby RAPE and MOLEST Scales as Measures of Cognitive Distortions With Civilly Committed Sexual Offenders. Sexual Abuse: Journal of Research and Treatment, 15(4), 237-249.

Bandura, A. (1977). Social learning theory. Englewood Cliffs, NJ: Prentice-Hall.

Becerra-García, J. A., García-León, A. et Egan, V. (2012). Childhood abuse history differentiates personality in sex offenders. Journal of Forensic Psychiatry \& Psychology, 23(1), 61-66.

Becerra-García, J. A., García-León, A., Muela-Martínez, J. A. et Egan, V. (2013). A controlled study of the Big Five personality dimensions in sex offenders, 
non-sex offenders and non-offenders: Relationship with offending behaviour and childhood abuse. Journal of Forensic Psychiatry \& Psychology, 24(2), 233-246.

Beech, A. R. (1998). A psychometric typology of child abusers. International Journal of Offender Therapy and Comparative Criminology, 42(4), 319-339.

Beech, A. R., Fisher, D. D. et Thornton, D. (2003). Risk assessment of sex offenders. Professional Psychology: Research and Practice, 34(4), 339-352.

Beech, A. R. et Ford, H. (2006). The relationship between risk, deviance, treatment outcome and sexual reconviction in a sample of child sexual abusers completing residential treatment for their offending. Psychology, Crime e Law, 12(6), 685-701.

Blumenthal, S., Gudjonsson, G. et Burns, J. (1999). Cognitive distortions and blame attribution in sex offenders against adults and children. Child Abuse \& Neglect, 23(2), 129-143.

Boccaccini, M. T., Murrie, D. C., Hawes, S. W., Simpler, A. et Johnson, J. (2010). Predicting recidivism with the Personality Assessment Inventory in a sample of sex offenders screened for civil commitment as sexually violent predators. Psychological Assessment, 22(1), 142-148.

Bogaerts, S., Daalder, A., Vanheule, S., Desmet, M. et Leeuw, F. (2008). Personality disorders in a sample of paraphilic and nonparaphilic child molesters: A comparative study. International Journal of Offender Therapy and Comparative Criminology, 52(1), 21-30.

Browne, K. D., Foreman, L. et Middleton, D. (1998). Predicting treatment drop-out in sex offenders. Child Abuse Review, 7(6), 402-419.

Bumby, K. M. (1996). Assessing the cognitive distortions of child molesters and rapists: Development and validation of the MOLEST and RAPE scales. Sexual Abuse: A Journal of Research and Treatment, 8(1), 37-54.

Burn, M. F. et Brown, S. (2006). A review of the cognitive distortions in child sex offenders: An examination of the motivations and mechanisms that underlie the justification for abuse. Aggression and Violent Behavior, 11(3), 225-236.

Carvalho, J. et Nobre, P. (2013). Dynamic factors of sexual aggression: The role of affect and impulsiveness. Criminal Justice and Behavior, 40(4), 376-387.

Christensen, L. et Mendoza, J. L. (1986). A method of assessing change in a single subject: An alteration of the RC index. Behavior Therapy, 17(3), 305-308.

Cohen, J. (1992). A power primer. Psychological bulletin, 112(1), 155-159.

Cohen, J. (1998). Statistical Power Analysis for the Behavioral Sciences (second ed.) Hillsdale, N.J.: L. Erlbaum Associates

Cohen, L. J., McGeoch, P. G., Watras-Gans, S., Acker, S., Poznansky, O., Cullen, K.,... Galynker, I. (2002). Personality impairment in male pedophiles. Journal of Clinical Psychiatry, 63(10), 912-919.

Collin-Vézina, D., Daigneault, I. et Hébert, M. (2013). Lessons learned from child sexual abuse research: Prevalence, outcomes, and preventive strategies. Child and Adolescent Psychiatry and Mental Health, 7(22), 1-9.

Costa, P. T. et McCrae, R. R. (1992). NEO Personality Inventory-Revised (NEOPI-R) and NEO Five-Factor Inventory (NEO-FFI) Professional Manual. Odessa, FL: Psychological Assessment Resources. 
Craig, L. A., Thornton, D., Beech, A. R. et Browne, K. D. (2007). The relationship of statistical and psychological risk markers to sexual reconviction in child molesters. Criminal Justice and Behavior, 34(3), 314-329.

Craissati, J. et Beech, A. (2001). Attrition in a community treatment program for child sexual abusers. Journal of Interpersonal Violence, 16(3), 205-221.

Dennison, S. M., Stough, C. et Birgden, A. (2001). The big 5 dimensional personality approach to understanding sex offenders. Psychology, Crime er Law, 7(3), 243-261.

Douglas, E. M. et Finkelhor, D. (2005). Childhood sexual abuse fact sheet. Durham, $\mathrm{NH}$ : Crimes against Children Research Center.

Egan, V., Kavanagh, B. et Blair, M. (2005). Sexual Offenders Against Children: The Influence of Personality and Obsessionality on Cognitive Distortions. Sexual Abuse: Journal of Research and Treatment, 17(3), 223-240.

Finkelhor, D., Hotaling, G., Lewis, I. A. et Smith, C. (1990). Sexual abuse in a national survey of adult men and women: Prevalence, characteristics, and risk factors. Child Abuse $\mathbb{O}$ Neglect, 14, 19-28.

Flores, J., Laforest, J. et Joubert, K. (2016). La violence vécue par les Québécois avant l'âge de 16 ans et la santé à l'âge adulte: quels sont les liens? Québec: Institut de la statistique du Québec.

Furniss, T. H. (1987). An integrated treatment approach to child sexual abuse in the family. Children $\sim$ Society, 1(2), 123-135.

Gannon, T. A., Ward, T., Beech, A. R. et Fisher, D. (2007). Aggressive offenders' cognition: Theory, research and practice. New York, NY: John Wiley \& Sons Ltd.

Goldberg, L. R. (1990). An alternative "description of personality": The BigFive factor structure. Journal of Personality and Social Psychology, 59(6), 12161229.

Grønnerød, C., Grønnerød, J. S. et Grøndahl, P. (2015). Psychological treatment of sexual offenders against children: A meta-analytic review of treatment outcome studies. Trauma, Violence, \& Abuse, 16(3), 280-290.

Guay, J.-P., Proulx, J. et Ouimet, M. (2001). L'estimation du niveau de concordance de trois modèles classificatoires d'agresseurs sexuels d'enfants: problèmes pratiques et implications théoriques. [Study of the level of agreement between three classification models of child sexual abusers : practical problems and theoretical implications.]. Canadian Journal of Criminology, 43(3), 357-384.

Hanson, R. K., Gizzarelli, R. et Scott, H. (1994). The attitudes of incest offenders: Sexual entitlement and acceptance of sex with children. Criminal Justice and Behavior, 21(2), 187-202.

Hanson, R. K., Gordon, A., Harris, A. J., Marques, J. K., Murphy, W., Quinsey, V. L. et Seto, M. C. (2002). First report of the collaborative outcome data project on the effectiveness of psychological treatment for sex offenders. Sexual Abuse: A Journal of Research and Treatment, 14(2), 169-194.

Hanson, R. K. et Harris, A. J. R. (2000). Where should we intervene? : Dynamic predictors of sexual assault recidivism. Criminal Justice and Behavior, $27(1), 6-35$. 
Hanson, R. K. et Morton-Bourgon, K. E. (2009). The accuracy of recidivism risk assessments for sexual offenders: A meta-analysis of 118 prediction studies. Psychological Assessment, 21(1), 1-21.

Hanson, R. K. et Thornton, D. (1999). Static 99: Improving actuarial risk assessments for sex offenders (Vol. 2). Ottawa, Ontario: Solicitor General Canada.

Hébert, M., Tourigny, M., Cyr, M., McDuff, P. et Joly, J. (2009). Prevalence of childhood sexuel abuse and timing of disclosure in a representative sample of adults from Quebec. The Canadian Journal of Psychiatry, 54(9), 631-636.

Helmus, L., Hanson, R. K., Babchishin, K. M. et Mann, R. E. (2013). Attitudes supportive of sexual offending predict recidivism: A meta-analysis. Trauma, Violence, $\mathfrak{O}$ Abuse, 14(1), 34-53.

Howitt, D. et Sheldon, K. (2007). The role of cognitive distortions in paedophilic offending: Internet and contact offenders compared. Psychology, Crime or Law, 13(5), 469-486.

Hunter Jr, J. A. et Figueredo, A. J. (1999). Factors associated with treatment compliance in a population of juvenile sexual offenders. Sexual Abuse:Journal of Research and Treatment, 11(1), 49-67.

Jacobson, N. S., Follette, W. C. et Revenstorf, D. (1984). Psychotherapy outcome research: Methods for reporting variability and evaluating clinical significance. Behavior Therapy, 15(4), 336-352.

Jacobson, N. S. et Truax, P. (1991). Clinical significance: a statistical approach to defining meaningful change in psychotherapy research. Journal of Consulting and Clinical Psychology, 59(1), 12.

Jespersen, A. F., Lalumière, M. L. et Seto, M. C. (2009). Sexual abuse history among adult sex offenders and non-sex offenders: A meta-analysis. Child Abuse \& Neglect, 33(3), 179-192.

Långström, N., Sjöstedt, G. et Grann, M. (2004). Psychiatric Disorders and Recidivism in Sexual Offenders. Sexual Abuse: Journal of Research and Treatment, 16(2), 139-150.

Listwan, S. J., Van Voorhis, P. et Ritchey, P. N. (2007). Personality, Criminal Behavior and Risk Assessment: Implications for Theory and Practice. Criminal Justice and Behavior, 34(1), 60-75.

Lösel, F. et Schmucker, M. (2005). The effectiveness of treatment for sexual offenders: A comprehensive meta-analysis. Journal of Experimental Crimino$\log y, 1(1), 117-146$.

Madsen, L., Parsons, S. et Grubin, D. (2006). The relationship between the five-factor model and DSM personality disorder in a sample of child molesters. Personality and Individual Differences, 40(2), 227-236.

Mandeville-Norden, R., Beech, A. R. et Hayes, E. (2008). Examining the effectiveness of a UK community-based sexual offender treatment programme for child abusers. Psychology, Crime $\mho ⿱ 乛 ⿻ 上 丨$ Law, 14(6), 493-512.

Mann, R., Webster, S., Wakeling, H. et Marshall, W. L. (2007). The measurement and influence of child sexual abuse supportive beliefs. Psychology, Crime or Law, 13(5), 443-458.

Marshall, W. L. et Barbaree, H. E. (1988). An outpatient treatment program for child molesters. Annals of the New York Academy of Sciences, 528, 205-214. 
Marshall, W. L., Barbaree, H. E. et Christophe, D. (1986). Sexual offenders against female children: Sexual preferences for age of victims and type of behaviour. Canadian Journal of Behavioural Science, 18(4), 424-439.

Marshall, W. L., Hamilton, K. et Fernandez, Y. (2001). Empathy deficits and cognitive distortions in child molesters. Sexual Abuse: A Journal of Research and Treatment, 13(2), 123-130.

Marziano, V., Ward, T., Beech, A. R. et Pattison, P. (2006). Identification of five fundamental implicit theories underlying cognitive distortions in child abusers: A preliminary study. Psychology, Crime \& Law, 12(1), 97-105.

Mihailides, S., Devilly, G. J. et Ward, T. (2004). Implicit cognitive distortions and sexual offending. Sexual Abuse: Journal of Research and Treatment, 16(4), 333-350.

Miner, M. H. et Dwyer, S. M. (1995). Analysis of dropouts from outpatient sex offender treatment. Journal of Psychology or Human Sexuality, 7(3), 77-93.

Moulden, H. M. (2008). Social competence and sexual aggression: Social intelligence, cognitive distortions, and victim empatby in men who sexually offend against children. (Thèse de doctorat, Université d'Ottawa). Repéré à http://www.ruor. uottawa.ca/bitstream/10393/29583/1/NR48408.PDF

Murphy, W. D. (1990). Assessment and modification of cognitive distortions in sex offenders. Dans W. L. Marshall, D. R. Laws et H. E. Barbaree (dir.), Handbook of sexual assault: Issues, theories, and treatment of the offender (p. 331342). New York, NY: Plenum Press.

Murphy, W. D. (2004). Management and Treatment of the Adult Sexual Offender (W. D. Murphy, Trans.). Dans B. J. Cling (dir.), Sexualized violence against women and children: A psychology and law perspective (p. 217-244). New York, NY: Guilford Press.

Muthén, L. K. et Muthén, B. O. (1998/2016). Mplus user's guide. Los Angeles, CA: Muthén \& Muthén.

Nunes, K. L., Babchishin, K. M. et Cortoni, F. (2011). Measuring treatment change in sex offenders: Clinical and statistical significance. Criminal Justice and Behavior, 38(2), 157-173.

Nunes, K. L. et Cortoni, F. (2008). Dropout from sex-offender treatment and dimensions of risk of sexual recidivism. Criminal Justice and Behavior, 35(1), 24-33.

Ogrodnik, L. (2010). Les enfants et les jeunes victimes de crimes violents déclarés par la police, 2008. Ottawa, Ontario: Statistique Canada.

Olver, M. E., Kingston, D. A., Nicholaichuk, T. P. et Wong, S. C. P. (2014). A psychometric examination of treatment change in a multisite sample of treated Canadian federal sexual offenders. Law and Human Behavior, 38(6), 544-559.

Olver, M. E. et Wong, S. (2011). Predictors of sex offender treatment dropout: Psychopathy, sex offender risk, and responsivity implications. Psychology, Crime $\mathfrak{*}$ Law, 17(5), 457-471.

Phelan, P. (1995). Incest and its meaning: The perspectives of fathers and daughters. Child Abuse or Neglect, 19(1), 7-24. 
Priest, R. et Smith, A. (1992). Counseling adult sex offenders: Unique challenges and treatment paradigms. Journal of Counseling $\mathcal{O}$ Development, 71(1), 27-32.

Randall, P., Carr, A., Dooley, B. et Rooney, B. (2011). Psychological characteristics of Irish clerical sexual offenders. The Irish Journal of Psychology, 32(12), 4-13.

Robertiello, G. et Terry, K. J. (2007). Can we profile sex offenders? A review of sex offender typologies. Aggression and Violent Behavior, 12(5), 508-518.

Roberts, B. W., Walton, K. E. et Viechtbauer, W. (2006). Patterns of mean-level change in personality traits across the life course: A meta-analysis of longitudinal studies. Psychological bulletin, 132(1), 1-25.

Robins, R. W., Fraley, R. C., Roberts, B. W. et Trzesniewski, K. H. (2001). A longitudinal study of personality change in young adulthood. Journal of Personality, 69(4), 617-640.

Sabourin, S. et Lussier, Y. (1992). Traduction française de l'inventaire de personnalité NEO-FFI. Manuscrit non publié.

Scott, R. L. et Stone, D. A. (1986). MMPI profile constellations in incest families. Journal of Consulting and Clinical Psychology, 54(3), 364-368.

Segal, Z. L. et Stermac, L. E. (1990). The role of cognition in sexual assault. Dans L. E. Marshall, D. R. Laws et H. E. Barbaree (dir.), Handbook of sexual assault: Issues, theories, and treatment of the offender (p. 161-174). New York, NY : Plenum Press.

Seto, M. (2007). Pedophilia and sexual offending against children: Theory, assessment, and treatment. Washington, DC: American Psychological Association.

Strassberg, D. S., Eastvold, A., Wilson Kenney, J. et Suchy, Y. (2012). Psychopathy among pedophilic and nonpedophilic child molesters. Child Abuse $\mathfrak{F}$ Neglect, 36(4), 379-382.

Tourigny, M., Daigneault, I., Hébert, M. et Wright, J. (2005). Portrait des signalements pour abus sexuels faits aux Directeurs et Directrices de la Protection de la jeunesse du Québec. Montréal, Québec: CRIPCAS.

Trocmé, N., MacLaurin, B., Fallon, B., Daciuk, J., Billingsley, D., Tourigny, M.,... McKenzie, B. (2001). Étude canadienne sur l'incidence des signalements de cas de violence et de négligence envers les enfants (ECI-1998): Rapport final. Ottawa, Ontario: Ministre de Travaux publics et Services gouvernementaux Canada.

Vaillancourt-Morel, M. P., Godbout, N., Sabourin, S., Briere, J., Lussier, Y. et Runtz, M. (2016). Adult sexual outcomes of child sexual abuse vary according to relationship status. Journal of Marital and Family Therapy, 42(2), 341-356.

Ward, T., \& Keenan, T. (1999). Child molesters' implicit theories. Journal of Interpersonal Violence, 14(8), 821-838.

Wright, J., \& Sabourin, S. (1985). L'intervention auprès du couple: Diagnostic et traitement. Montréal, Québec: Les Éditions Consultation.

Yesberg, J. A. et Polaschek, D. L. L. (2015). Assessing dynamic risk and protective factors in the community: Examining the validity of the Dynamic Risk Assessment for Offender Re-entry. Psychology, Crime $\leftarrow$ Law, 21(1), 80-99. 


\title{
Personality and cognitive distortions in sexual offenders: longitudinal changes after treatment for child sexual abuse
}

\begin{abstract}
Although there is no clear typology of the characteristics of child molesters, the scientific literature highlights cognitive distortions and a constellation of maladaptive personality traits involved in the development and maintenance of deviant sexuality. As these factors also contribute to the development of dysfunctional sexual behaviors and to the risk of recidivism, they may be key targets for intervention. The current study examines changes in cognitive distortions and personality traits in men being treated for child sexual abuse. The sample was composed of 134 intrafamilial child molesters enrolled in an evaluation and treatment program for sexual abuse. Participants were assessed three times: at their admission to the program and after the first and second year of treatment. Results suggest significant decreases in cognitive distortions as well as decreases in neuroticism and increases in extraversion. At the individual level, a meaningful proportion of men showed improvements on most of the variables assessed. These findings support the plasticity of personality and cognitive distortions in child molesters and provide evidence for the effectiveness of the program.
\end{abstract}

KEYWORDS - Child sexual abuse, cognitive distortions, personality, treatment.

\section{Personalidad y distorsiones cognitivas de los agresores sexuales. Cambios longitudinales después de un tratamiento por agresión sexual hacia los niños}

RESUMEN - Aunque no existe una tipología definitiva de las características de los agresores sexuales de niños, la documentación científica revela la presencia de distorsiones cognitivas y de rasgos de personalidad implicados en la etiología y en el mantenimiento de comportamientos sexuales desviados. Dichos factores están igualmente asociados al riesgo de reincidencia y constituyen objetivos de tratamiento de primera importancia. El presente estudio tiene como objetivo examinar la evolución de las distorsiones cognitivas y de los rasgos de personalidad de hombres en terapia por agresiones sexuales a niños. La muestra está compuesta por 134 agresores sexuales intrafamiliares reclutados en su entrada al Programa de evaluación y de tratamiento de situaciones de agresiones sexuales (PÉTAS). Éstos son evaluados en tres oportunidades, es decir, al comienzo del PÉTAS, y también al final del premier y del segundo año de tratamiento. Los resultados muestran bajas significativas del conjunto de distorsiones cognitivas de naturaleza sexual, una disminución de la neurosis y una aumentación de la extraversión entre los tiempos de medida. En el plano individual, una proporción importante de los hombres presentan mejoras sobre la mayoría de las variables medidas. Estos resultados apoyan la maleabilidad de las distorsiones cognitivas y de ciertos rasgos de personalidad, y apoyan la eficacidad del PÉTAS.

PALABRAS CLAVE - Agresores sexuales, distorsiones cognitivas, personalidad, tratamiento. 